\title{
A systematic diagnostic evaluation combined with an internet-based self-management support system for patients with asthma or COPD
}

This article was published in the following Dove Press journal: International Journal of COPD

\author{
Amanda $R$ van Buul' \\ Thomas S Wildschut ${ }^{1,2}$ \\ Tobias N Bonten ${ }^{1,2}$ \\ Marise J Kasteleyn ${ }^{1,2}$ \\ Annelies M Slats' \\ Niels H Chavannes ${ }^{2}$ \\ Christian Taube ${ }^{1,3}$
}

'Department of Pulmonology, Leiden University Medical Center, Leiden, the Netherlands; ${ }^{2}$ Department of Public Health and Primary Care, Leiden University Medical Center, Leiden, the Netherlands; ${ }^{3}$ Department of Pulmonary Medicine, West German Lung Center, Essen University Hospital, Ruhrlandklinik, University Duisburg-Essen, Essen, Germany
Correspondence: Amanda R van Buul Department of Pulmonology, postzoneC2-R, Leiden University Medical Center, Albinusdreef 2, Postbus 9600 2300 RC Leiden, the Netherlands

$\mathrm{Tel}+3$ I 7| 5298726

Fax +3I 7I 5266927

Email a.r.van_buul@lumc.nl
Introduction: An (inter)national systematic approach for patients with asthma COPD referred to secondary care is lacking. Therefore, a novel systematic approach was designed and tested in clinical practice.

Methods: This was a retrospective observational study of data from the electronic record system of the Leiden University Medical Center. Asthma and COPD patients were included if they were evaluated with a novel systematic approach or if they had a new record for asthma or COPD and received usual care. The novel systematic approach consisted of a predefined diagnostic evaluation combined with an optional internet-based self-management support system. Diagnostic tests, final diagnosis, lifestyle advices, symptoms and individual care plans in the electronic records, number of patients referred back to primary care, and time to referral back to primary care were compared between the systematic approach and usual care groups using $t$-tests and chi-squared tests.

Results: A total of 125 patients were included, of which $22(21.4 \%)$ were evaluated with the systematic approach. Mean $( \pm \mathrm{SD})$ age was $48.8( \pm 18.4)$ years and $59.2 \%$ were women. Mean $( \pm \mathrm{SD})$ number of diagnostic tests was higher in the systematic approach group compared with the usual care group ( $7.6 \pm 1.0$ vs $5.5 \pm 1.8, P<0.001)$. Similarly, in the systematic approach group, more lifestyle advices ( $81.8 \%$ vs $29.1 \%$ ), symptom scores ( $95.5 \%$ vs $21.4 \%$ ), and individual care plans $(50.0 \%$ vs $7.8 \%)$ were electronically recorded $(P<0.001)$, and more patients were referred back to primary care $(81.8 \%$ vs $56.3 \%, P=0.03)$. There were no differences in the final diagnoses and time to referral back.

Conclusion: Our study suggested that not all tests that were included in the systematic approach are regularly needed in the diagnostic work-up. In addition, a designated systematic approach stimulates physicians to record lifestyle advices, symptoms, and individual care plans. Thus, this approach could increase the number of patients referred back to primary care.

Keywords: asthma, COPD, diagnostics, eHealth, outpatient clinic, systematic approach

\section{Introduction}

Asthma and COPD are common obstructive lung diseases. It is important to differentiate between asthma, COPD, and other (lung) diseases to phenotype these diseases even if the asthma/COPD is not severe as well as to design individual care plans. International guidelines have suggested on the parameters that should be assessed to evaluate asthma and COPD. ${ }^{1-3}$ However, the guidelines do not provide suggestions on the additional tests that should be necessarily added to decrease the likelihood of other diagnoses, nor on the tests that should preferably be performed to identify treatable traits. A review showed that the use of care pathways results in a reduction of costs, improved quality 
of life, reduced number of complications, increased patient satisfaction, improved communication between doctors and nurses, and reduced time that healthcare providers spent carrying out paperwork. ${ }^{4}$ However, for unknown reasons, a uniform international systematic approach for assessment of asthma and COPD patients referred to secondary care is lacking. Therefore, a diagnostic approach has been developed for asthma and COPD patients in the Netherlands. This diagnostic approach includes functional tests in addition to additional tests to acquire detailed insights into symptoms, functional limitation, and quality of life, ultimately to create a personalized treatment plan. ${ }^{5}$ Patients are involved in this treatment plan in formulating their own treatment goals. However, it is thought that patient involvement could be increased by the use of eHealth. The previous diagnostic approach has been successfully implemented. However, the effects of the use of this diagnostic approach on the number of diagnostics, final diagnoses, referrals back to primary care, and long-term outcomes have not been evaluated yet. In addition, eHealth could be used as a method to integrate the individual care plans. ${ }^{6}$ Therefore, in the LUMC, a novel systematic approach was developed that consisted of a predefined diagnostic evaluation combined with an optional internet-based self-management support system. Since March 2016, patients with (suspected) asthma or COPD who were referred by a GP to secondary pulmonary care were systematically evaluated with this systematic approach. The primary aim of this study was to determine whether there was a difference between the systematic approach and usual care in terms of type of diagnostic tests, number of diagnostic tests, final diagnosis, recorded lifestyle advices, symptoms and individual care plans in the electronic records, number of patients referred back to primary care, and time to referral back to primary care. The secondary aim of this study was to evaluate the number of patients who used the internet-based self-management support system. We hypothesize that a systematic approach could lead to a more specified diagnosis, more lifestyle advices, symptoms and individual care plans that are recorded in the electronic records, and more and faster referral back to primary care.

\section{Materials and methods Diagnostic pathway}

Since March 2016, a novel systematic approach to evaluate patients with (suspected) asthma or COPD was released at the outpatient clinic of the department of pulmonology at the LUMC. A pulmonologist who was not involved in the study assessed the urge of the GP referrals. Patients with urgent complaints were scheduled at the outpatient clinic for acute respiratory complaints. The other patients were scheduled either within the novel systematic approach or by usual outpatient clinic care, depending on the first possibility at the outpatient clinic. With usual care, physicians decided which diagnostic tests to be performed based on outcomes of the clinical history and personal preferences. The systematic approach consisted of a predefined systematic diagnostic evaluation combined with an optional internetbased self-management support system. There were two or three visits, or more if there was a medical reason for additional diagnostic tests (Figure 1). During the first visit, a nurse practitioner obtained general information, vital parameters, and evaluated inhaler technique. The same day, lung function tests (spirometry before and after bronchodilation and DLCO) were performed. $\mathrm{Fe}_{\mathrm{NO}}$ was measured and a chest X-ray, ECG, and laboratory tests (including hemoglobin, erythrocyte sedimentation rate, white blood cell count with differential, sodium, potassium, creatinine, radioallergosorbent test, thyroid stimulating hormone, N-terminal pro B-type natriuretic peptide, total immunoglobulin E, 25-hydroxyvitamin D, and alpha 1-antitrypsin) were performed. The nurse informed the patients about the internet-based self-management support system in the form of "PatientCoach". PatientCoach has been developed by the LUMC specifically for patients with chronic diseases and contains information about asthma and COPD, self-monitoring, an individual care plan, and e-visits. ${ }^{7}$ The GP can also access PatientCoach. If a patient agreed to use PatientCoach, he or she was asked to complete the Nijmegen Clinical Screening Instrument ${ }^{8}$ and asthma control questionnaire ${ }^{9}$ or the clinical COPD questionnaire ${ }^{10}$ at home. In addition, patients formulated their own personal treatment goals. During the second visit, the nurse practitioner discussed the outcomes of the questionnaires and the personal goals. The second visit was canceled if the patients refused to use PatientCoach.

During the last planned visit, a pulmonologist or a pulmonology resident discussed the outcomes of the diagnostic tests with the patient and an individual care plan was created. If a patient used PatientCoach, the individual care plan was integrated in PatientCoach. If additional diagnostic tests, eg, chest CT scan or sputum cultures, were needed, patients were scheduled for a fourth or later follow-up visit. If no more visits in the hospital were necessary, patients were referred back to primary care. The GP and the patient still had access to PatientCoach, enabling the continuation of eHealth in further care.

\section{Study design}

This was a retrospective observational study of real-life data that were retrieved from the electronic record system of the 


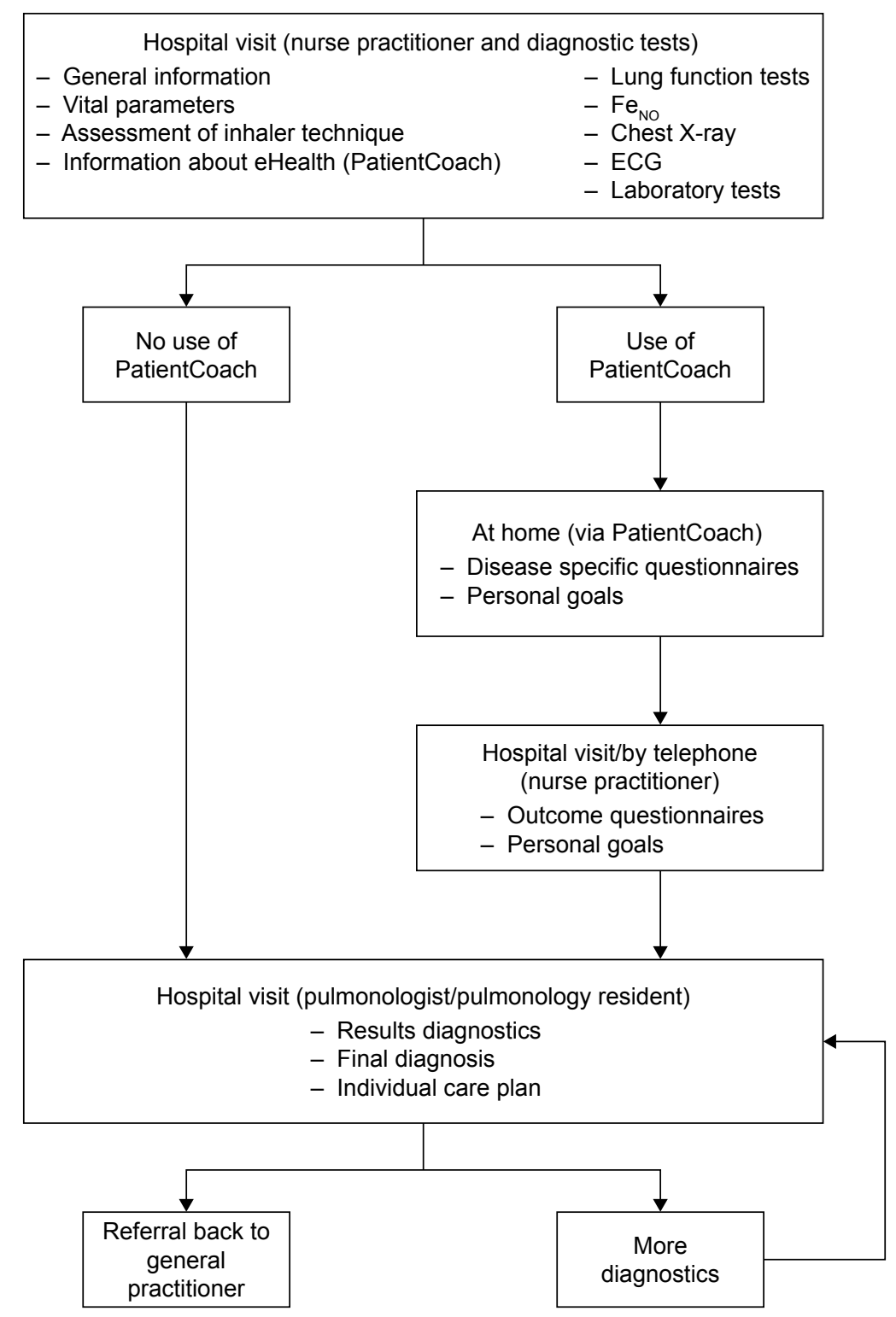

Figure I Novel systematic approach for asthma and COPD at the LUMC.

Abbreviations: $\mathrm{ECG}$, electrocardiography; $\mathrm{Fe}_{\mathrm{NO}}$, Fractional exhaled nitric oxide; $\mathrm{LUMC}$, Leiden University Medical Center.

LUMC. Data were collected between November 2017 and January 2018 from patients who attended the outpatient clinic of the LUMC for the first time between March 2016 and July 2017. The medical ethical committee of the LUMC waived the need for ethical approval due to the retrospective nature of the study. Consequently, the need for informed consent was not applicable.

\section{Study population}

Patients were included if they were evaluated with the systematic approach or if they had a new record for asthma or COPD and were evaluated by usual outpatient clinic care. Patients were included if they attended the outpatient clinic of the LUMC for the first time between March 2016 and July
2017. Patients were excluded if they attended the dyspnea clinic of the LUMC, if they had a previous record of asthma or COPD at the department of pulmonology at the LUMC, if they were solely admitted to the clinical ward; if they were not referred by a GP, if the first visit was at the emergency room or in the clinical ward, if the reason for referral was not asthma or COPD, or if the patient did not show up at the outpatient visits.

\section{Data collection}

Baseline characteristics were collected from the electronic records from the first visit at the department of pulmonology at the LUMC. Type of diagnostic tests, number of diagnostic tests, final diagnosis, lifestyle advices, symptoms and 
individual care plans in the electronic records, number of patients referred back to primary care, and time to referral back to primary care were collected from the electronic records as primary outcomes. The secondary outcome was the number of patients who used PatientCoach.

Diagnostic tests, needed for the work-up to the final diagnosis, were evaluated. The type of diagnostic tests that were assessed included laboratory tests, lung-specific laboratory tests (including arterial blood gas analysis, alpha-1 antitrypsin, and radioallergosorbent test), spirometry, histamine provocation test, $\mathrm{Fe}_{\mathrm{NO}}$, DLCO, chest X-ray, CT (including pulmonary CT angiography), bronchoscopy, sputum cultures, six-minute walking test, and ECG. The final diagnosis that was registered in the electronic records was evaluated. Predefined diagnoses were asthma, COPD, a combination of asthma and COPD, another pulmonary disease, no pulmonary disease, obesity-related symptoms, or no diagnosis. If the final diagnosis was asthma and/or COPD, the phenotype was evaluated. Predefined phenotypes included allergic, nonallergic, late-onset asthma, asthma with fixed airflow limitation, and asthma with obesity. ${ }^{1}$ Alpha-1 antitrypsin deficiency, emphysema/hyperinflation, and frequent exacerbators ${ }^{11}$ (including the GOLD group B and $\mathrm{D}^{2}$ ) were predefined phenotypes in COPD. The number of reported symptom scores, lifestyle advices, and individual care plans were collected from the electronic records. The number of patients who were referred back to primary care was collected. The number of days between referral and first visit, between first visit and final diagnosis, between first visit and the latest diagnostic test, and between first visit and referral back to primary care were calculated.

The number of patients who logged in at least once in PatientCoach was collected from the electronic records.

\section{Statistical analysis}

Patients with missing data on the key variables were excluded $(\mathrm{N}=2)$. Descriptive data were reported as percentages and mean values $\pm \mathrm{SD}$ for continuous variables. To compare means between the systematic approach and usual care groups for continuous baseline characteristics as age and BMI, unpaired $t$-tests were used. To compare percentages between the systematic approach and usual care groups for categorical baseline characteristics as percentage women and smoking status, chi-squared tests were used. To compare percentages of performed diagnostic tests between the systematic approach and usual care groups, chi-squared tests were used. To compare the mean time between referral and other time points and the mean number of performed diagnostic tests, between the systematic approach and usual care groups, unpaired $t$-tests were used. To compare percentages of final diagnosis as asthma and COPD, electronically recorded lifestyle advices, electronically recorded symptoms scores, electronically recorded individual care plans, and referrals back to primary care, between the systematic approach and usual care groups, chi-squared tests were used.

Since the use of PatientCoach was optional and a part of patients refused to use it, the number of patients who logged in at least once in PatientCoach was collected. The number of patients who logged in at least once in PatientCoach was expressed as percentage of the total number of patients who were included in the diagnostic approach. To compare means between patients in the systematic approach group who used PatientCoach and who did not for continuous baseline characteristics, unpaired $t$-tests were used. To compare percentages between patients in the systematic approach group who used PatientCoach and who did not, for categorical baseline characteristics, chi-squared tests were used. Furthermore, a sensitivity analysis was performed to evaluate the differences in primary outcomes between the patients in the usual care and those in the diagnostic approach who used PatientCoach. All analysis were conducted using SPSS version 23.0.

\section{Ethics statement}

The medical ethical committee of the LUMC waived the need for ethical approval due to the retrospective nature of the study. Consequently, the need for informed consent was not applicable. Data that were entered in the database were de-identified.

\section{Results \\ General outcomes}

In total, 125 out of 608 patients were included in the analysis (Figure 2), of which 22 (21.4\%) were evaluated with the systematic approach. Baseline characteristics of all included patients are presented in Table 1 . In total, $67.2 \%$ of patients were referred for asthma. Mean $( \pm \mathrm{SD})$ age was $48.8( \pm 18.4)$ years and $59.2 \%$ of patients were women. There were no significant differences in baseline characteristics between patients who were evaluated with the systematic approach compared with usual care.

\section{Diagnostic tests}

The most frequently performed diagnostic test was spirometry $(97.6 \%)$ (Table 2$)$. The mean $( \pm \mathrm{SD})$ number of diagnostic tests was significantly higher in the systematic approach group compared with the usual care group (7.6 \pm 1.0 vs $5.5 \pm 1.8)$. Laboratory tests, lung-specific laboratory tests, $\mathrm{Fe}_{\mathrm{NO}}$, DLCO, chest X-rays, and ECG were more frequently performed in the systematic approach group than in the usual care group. 


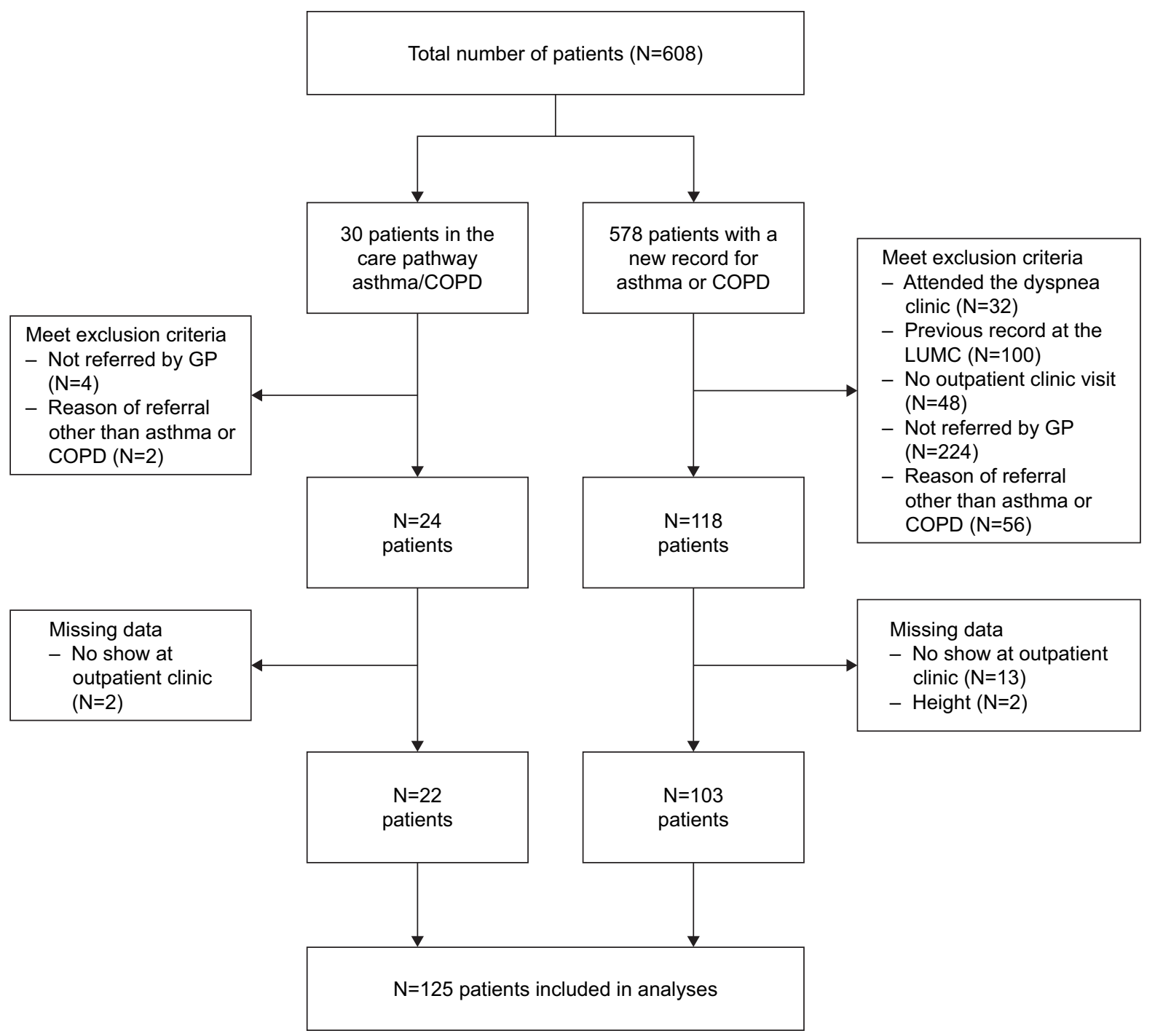

Figure 2 Flow diagram.

Abbreviations: GP, general practitioner; LUMC, Leiden University Medical Center.

\section{Final diagnosis and registered symptom scores, lifestyle advices, and individual care plans}

Most patients were finally diagnosed with asthma (53.6\%), COPD (25.6\%), or no pulmonary disease $(9.8 \%)$. There were no differences in final diagnosis and phenotypes between the systematic approach and usual care groups (Table 3). In the systematic approach group, more lifestyle advices $(81.8 \%$ vs $29.1 \%$ ), symptom scores (95.5\% vs $21.4 \%$ ), and individual care plans $(50.0 \%$ vs $7.8 \%)$ were electronically registered compared with the usual care group (all $P<0.001$ ).

\section{Time to final diagnosis and referral back to primary care}

There were differences in the number of days between referral and first visit (difference 4.4 days), between first visit and latest diagnostic test (difference 22.7 days), and between first visit and final diagnosis (difference 34.1 days) between the systematic approach and usual care groups. However, these differences did not reach significance (Table 4). More patients in the systematic approach group were referred back to primary care compared with the usual care group $(81.8 \%$ vs $56.3 \%, P=0.03)$.

\section{Use of PatientCoach}

In the systematic approach group, 14 out of 22 patients (63.6\%) logged in at least once on PatientCoach. Patients who used PatientCoach were significantly younger and had less cardiovascular comorbidities than those who did not use PatientCoach. Reasons to refuse the use of PatientCoach were not having a computer $(\mathrm{N}=2)$, lack of computer skills $(\mathrm{N}=4)$, definitely no desire to use PatientCoach without a further reason $(\mathrm{N}=1)$, and for one patient the reason was not applicable. 
Table I Baseline characteristics

\begin{tabular}{|c|c|c|c|c|}
\hline Characteristics & $\begin{array}{l}\text { Total } \\
\text { group } \\
(\mathbf{N}=\mid 25)\end{array}$ & $\begin{array}{l}\text { Usual care } \\
(\mathrm{N}=103)\end{array}$ & $\begin{array}{l}\text { Systematic } \\
\text { approach } \\
(\mathbf{N}=22)\end{array}$ & $\begin{array}{l}\text { Difference } \\
\text { (P-value) }\end{array}$ \\
\hline Age in years (mean, SD) & $48.8(18.4)$ & $48.2(18.2)$ & $51.8(19.5)$ & 0.41 \\
\hline Sex, n (\% women) & $74(59.2)$ & $58(56.3)$ & $16(72.7)$ & 0.16 \\
\hline $\mathrm{BMI}$ in kg/m (mean, SD) & $27.0(6.8)$ & $26.7(6.7)$ & $28.2(7.6)$ & 0.37 \\
\hline Cardiovascular comorbidities, n (\% yes) & $28(22.4)$ & $23(22.3)$ & $5(22.7)$ & 0.97 \\
\hline Reason of referral, $n$ (\% asthma) & $84(67.2)$ & $69(67.0)$ & $15(68.2)$ & 0.91 \\
\hline \multicolumn{5}{|l|}{ Smoker status } \\
\hline Never smoker, n (\% yes) & $55(44.0)$ & $43(41.7)$ & $12(54.5)$ & 0.27 \\
\hline Former smoking, n (\% yes) & $44(35.2)$ & $39(37.9)$ & $5(22.7)$ & 0.18 \\
\hline Current smoker, $\mathrm{n}(\%$ yes) & $26(20.8)$ & $21(20.4)$ & $5(22.7)$ & 0.81 \\
\hline \multicolumn{5}{|l|}{ Medication use at baseline } \\
\hline SABA, n (\% yes) & $73(58.4)$ & $57(55.3)$ & $16(72.7)$ & 0.13 \\
\hline LABA, n (\% yes) & $79(63.2)$ & $63(61.2)$ & $16(72.7)$ & 0.31 \\
\hline SAMA, n (\% yes) & $16(12.8)$ & $14(13.6)$ & $2(9.1)$ & 0.57 \\
\hline LAMA, n (\% yes) & $29(23.2)$ & $23(22.3)$ & $6(27.3)$ & 0.62 \\
\hline ICS, n (\% yes) & $90(72.0)$ & $73(70.9)$ & $17(77.3)$ & 0.54 \\
\hline LTRA, n (\% yes) & $9(7.2)$ & $6(5.8)$ & $3(13.6)$ & 0.20 \\
\hline Antihistamines, $\mathrm{n}$ (\% yes) & $36(28.8)$ & $28(27.2)$ & $8(36.4)$ & 0.39 \\
\hline
\end{tabular}

Abbreviations: ICS, inhaled corticosteroids; LABA, long-acting beta2-agonist; LAMA, long-acting muscarinic-antagonist; LTRA, leukotriene receptor antagonist; SABA, short-acting beta2-agonist; SAMA, short-acting muscarinic-antagonist.

\section{Sensitivity analysis}

After removing eight patients who attended the systematic approach, but refused the use of PatientCoach, the statistically significant differences between the systematic approach and usual care were still present (Tables S1 and S2).

\section{Discussion}

This study was conducted to evaluate a systematic approach combined with an optional internet-based self-management support system for asthma and COPD patients referred to secondary care. In the systematic approach group compared with the usual care group, mean number of diagnostic tests was higher; more lifestyle advices, symptom scores, and individual care plans were electronically recorded; and more patients were referred back to primary care. There were no differences in final diagnosis and time to referral back. More than half of patients were interested in an internet-based selfmanagement support system when it was offered in addition to a systematic approach.

To our knowledge, this is the first study that evaluated a systematic approach in combination with an optional internetbased self-management support system as a method to

Table 2 Diagnostic tests at the outpatient clinic for the evaluation of asthma and COPD

\begin{tabular}{|c|c|c|c|c|}
\hline Diagnostic tests & $\begin{array}{l}\text { Total } \\
\text { group } \\
(\mathbf{N}=\mid 25)\end{array}$ & $\begin{array}{l}\text { Usual care } \\
(\mathrm{N}=103)\end{array}$ & $\begin{array}{l}\text { Systematic } \\
\text { approach } \\
(\mathrm{N}=\mathbf{2 2})\end{array}$ & $\begin{array}{l}\text { Difference } \\
\text { (P-value) }\end{array}$ \\
\hline Laboratory tests, n (\% yes) & $108(86.4)$ & $86(83.5)$ & $22(100.0)$ & 0.040 \\
\hline Lung-specific laboratory tests, $n$ (\% yes) & $100(80.0)$ & $79(76.7)$ & $21(95.5)$ & 0.046 \\
\hline Spirometry, n (\% yes) & $122(97.6)$ & $100(97.1)$ & $22(100.0)$ & 0.42 \\
\hline Histamine provocation test, $\mathrm{n}$ (\% yes) & $40(32.0)$ & $34(33.0)$ & $6(27.3)$ & 0.60 \\
\hline $\mathrm{Fe}_{\mathrm{NO}}, \mathrm{n}(\%$ yes $)$ & $85(68.0)$ & $64(62.1)$ & $21(95.5)$ & 0.002 \\
\hline DLCO, n (\% yes) & $88(70.4)$ & $66(64.1)$ & $22(100.0)$ & 0.001 \\
\hline Chest X-ray, n (\% yes) & $97(77.6)$ & $76(73.8)$ & $21(95.5)$ & 0.027 \\
\hline Chest CT scan, $\mathrm{n}$ (\% yes) & $23(18.4)$ & $18(17.5)$ & $5(22.7)$ & 0.56 \\
\hline Bronchoscopy, n (\% yes) & I $(0.8)$ & I ( 1.0$)$ & $0(0.0)$ & 0.64 \\
\hline Sputum cultures, n (\% yes) & $46(19.2)$ & $18(17.5)$ & $6(27.3)$ & 0.29 \\
\hline Six-minute walking test, $\mathrm{n}$ (\% yes) & I $(0.8)$ & I (I.0) & $0(0.0)$ & 0.64 \\
\hline ECG, $n$ (\% yes) & $38(30.4)$ & $18(17.5)$ & $20(90.9)$ & $<0.001$ \\
\hline Number of diagnostic tests (mean, SD) & $5.8(1.9)$ & $5.5(1.8)$ & $7.6(1.0)$ & $<0.001$ \\
\hline
\end{tabular}

Notes: Bold values indicate statistical significance.

Abbreviations: $\mathrm{CT}$, computed tomography; DLCO, diffusing capacity for carbon monoxide; $\mathrm{ECG}$, electrocardiography; $\mathrm{Fe}_{\mathrm{NO}}$, fractional exhaled nitric oxide. 
Table 3 Final diagnosis, phenotypes, and treatment plans in the diagnostic pathway compared with usual care

\begin{tabular}{|c|c|c|c|c|}
\hline Final diagnosis and treatment & $\begin{array}{l}\text { Total } \\
\text { group } \\
(\mathbf{N}=\mid 25)\end{array}$ & $\begin{array}{l}\text { Usual care } \\
(\mathrm{N}=103)\end{array}$ & $\begin{array}{l}\text { Systematic } \\
\text { approach } \\
(\mathbf{N}=22)\end{array}$ & $\begin{array}{l}\text { Difference } \\
\text { (P-value) }\end{array}$ \\
\hline \multicolumn{5}{|l|}{ Final diagnosis } \\
\hline Asthma, n (\%) & $67(53.6)$ & $56(54.4)$ & II (50.0) & 0.71 \\
\hline Phenotyped, n (\% yes) & $57(85.1)$ & $46(82.1)$ & $11(100.0)$ & 0.13 \\
\hline Allergic asthma, ${ }^{\mathrm{a}} \mathrm{n}$ (\% yes) & $44(65.7)$ & $35(62.5)$ & $9(81.8)$ & 0.22 \\
\hline Nonallergic asthma, ${ }^{\mathrm{a}} \mathrm{n}$ (\% yes) & II (I6.4) & $9(16.1)$ & $2(18.2)$ & 0.86 \\
\hline Late-onset, ${ }^{\mathrm{a}} \mathrm{n}$ (\% yes) & $7(10.4)$ & $6(10.7)$ & I $(9.1)$ & 0.87 \\
\hline With fixed airflow limitation, ${ }^{a} \mathrm{n}$ (\% yes) & $4(6.0)$ & $4(7.1)$ & $0(0.0)$ & 0.36 \\
\hline Asthma with obesity, ${ }^{a}$ (\% yes) & $0(0.0)$ & $0(0.0)$ & $0(0.0)$ & NA \\
\hline COPD, n (\%) & $32(25.6)$ & $27(26.2)$ & $5(22.7)$ & 0.73 \\
\hline Phenotyped, n (\% yes) & $18(56.3)$ & $16(59.3)$ & $2(40.0)$ & 0.43 \\
\hline AAT deficiency, ${ }^{\mathrm{a}} \mathrm{n}$ (\% yes) & $0(0.0)$ & $0(0.0)$ & $0(0.0)$ & NA \\
\hline Emphysema/hyperinflation, ${ }^{\mathrm{a}} \mathrm{n}$ (\% yes) & $6(18.8)$ & $6(22.2)$ & $0(0.0)$ & 0.24 \\
\hline Frequent exacerbations, ${ }^{a} \mathrm{n}$ (\% yes) & $12(37.5)$ & $10(37.0)$ & $2(40.0)$ & 0.90 \\
\hline Combination of asthma and COPD, $\mathrm{n}(\%)$ & I $(0.8)$ & $\mathrm{I}(\mathrm{I} .0)$ & $0(0.0)$ & 0.64 \\
\hline Phenotyped, n (\% yes) & $\mathrm{I}(100.0)$ & I (I00.0) & $0(0.0)$ & NA \\
\hline Other pulmonary disease, $\mathrm{n}$ (\% yes) & $6(4.8)$ & $4(3.9)$ & $2(9.1)$ & 0.30 \\
\hline No pulmonary disease, $\mathrm{n}$ (\% yes) & $12(9.6)$ & $10(9.7)$ & $2(9.1)$ & 0.93 \\
\hline Obesity-related symptoms, n (\% yes) & $3(2.4)$ & $2(1.9)$ & $\mathrm{I}(4.5)$ & 0.47 \\
\hline No diagnosis, $n$ (\% yes) & $2(1.6)$ & $2(1.9)$ & $0(0.0)$ & 0.51 \\
\hline Lifestyle advices, n (\% yes) & $48(38.4)$ & $30(29.1)$ & I8 (8I.8) & $<0.00 \mathrm{I}$ \\
\hline Symptom scores, n (\% yes) & $43(34.4)$ & $22(21.4)$ & $21(95.5)$ & $<0.001$ \\
\hline Individual care plans, n (\% yes) & $19(15.2)$ & $8(7.8)$ & II (50.0) & $<0.00 \mathrm{I}$ \\
\hline
\end{tabular}

Notes: aPatients could be categorized in more than one phenotype. Bold values indicate statistical significance.

Abbreviation: AAT, alpha I-antitrypsin.

evaluate diagnosis and symptoms and to integrate individual care plans in secondary care pulmonology. More diagnostic tests were performed in the systematic approach compared to usual care in the present study. This could be expected since the diagnostic approach consisted of more diagnostic tests than are stated in the guidelines. Since there were no differences in the final diagnoses made by pulmonologists and the process was not faster, this suggests that not all tests included in the systematic approach are regularly needed in the diagnostic work-up. In another systematic approach that has been developed for asthma and COPD patients in the Netherlands, other standard diagnostic tests were used: capillary blood gas analyses, metronome-paced hyperventilation test, and physical activity assessment by accelerometry were performed in all patients; X-rays, $\mathrm{Fe}_{\mathrm{NO}}$, and allergic assessment were performed only in selected patients. ${ }^{5}$ Taking the results of the present study and the previous Delphi study into account, it can be suggested that lung-specific laboratory tests, DLCO, X-rays, $\mathrm{Fe}_{\mathrm{NO}}$, and an ECG should preferably be performed on indication and should not be included in a systematic approach in nonselected patients, since these additional tests did not lead to other diagnoses. This decision will decrease the number of diagnostic tests and thereby costs. On the other hand, it is possible that the additional diagnostic tests contributed more certainty to a diagnoses of asthma or COPD, resulting in more referrals back to primary care, as observed in our study. This follows the Dutch guidelines to refer patients back to primary care

Table 4 Time between referral, first visit, final diagnosis, latest diagnostic test, and referral back to primary care

\begin{tabular}{|c|c|c|c|c|}
\hline Time & $\begin{array}{l}\text { Total } \\
\text { group } \\
(\mathbf{N}=\mid 25)\end{array}$ & $\begin{array}{l}\text { Usual care } \\
(\mathbf{N}=103)\end{array}$ & $\begin{array}{l}\text { Systematic } \\
\text { approach } \\
(\mathbf{N}=22)\end{array}$ & $\begin{array}{l}\text { Difference } \\
\text { (P-value) }\end{array}$ \\
\hline From referral to first visit in days (mean, SD) & $37.7(23.6)$ & $38.4(24.6)$ & $34.0(17.8)$ & 0.43 \\
\hline From first visit to final diagnosis in days (mean, SD) & $68.3(88.0)$ & $72.3(91.7)$ & $49.6(66.9)$ & 0.28 \\
\hline From first visit to latest diagnostic test in days (mean, SD) & I 16.7 (I27.3) & 122.7 ( 128.7$)$ & $88.6(119.2)$ & 0.26 \\
\hline Referred back to primary care, n (\%) & $76(60.8)$ & $58(56.3)$ & $18(8 \mid .8)$ & 0.03 \\
\hline Time from first visit and referral to primary care in days (mean, SD) & I I4.I (97.7) & II $3.3(96.0)$ & 116.5 (I05.8) & 0.91 \\
\hline
\end{tabular}

Note: Bold values indicate statistical significance. 
when the diagnostic work-up is completed and patients are clinically stable. ${ }^{12}$ Another explanation of more referrals back to primary care might be the higher number of written care plans, since pulmonologists might believe that the care plans will support the GP and patients in the continuation of care. The present study shows that if physicians are directed to register individual care plans, this was done more frequently than with usual care. This is in line with a systematic review that the use of clinical pathways results in improved documentation. ${ }^{13}$ We think that more than $48.5 \%$ of patients in the usual care group did receive lifestyle advices and individual care plans, but these advises were given verbally and were not necessarily registered in the medical records. However, international guidelines recommend written care plans and not solely spoken arrangements. ${ }^{1,2}$ A designated pathway within an outpatient clinic could thus add to improve registration of individual care plans.

The internet-based self-management support system was accepted by $63.6 \%$ of patients, which is in line with a previous study that showed that $63 \%$ of patients would definitely or probably use an eHealth application if it was offered. ${ }^{14}$ In the present study, reasons to refuse eHealth were mostly computer (skill) related. A previous study showed that patients who do not use eHealth do not recognize the advantages of eHealth and should be convinced first. ${ }^{15}$ Patients who do have experience with the use of eHealth are positive. ${ }^{15}$ The use of PatientCoach may be lower than $63.6 \%$, because only the first login was evaluated, and it is known from previous research that some patients stop using the application. ${ }^{16}$ Probably, more patients would use PatientCoach when previously determined barriers such as sufficient functionalities to tailor PatientCoach and personal guidance are further optimized. ${ }^{17}$ An advantage of the use of eHealth is that patients considered eHealth as a possibility to take more responsibility in their own care. ${ }^{15}$

A strength of the present study was that the outcomes seem to be generalizable to all asthma and COPD patients referred to secondary care since we included nonselected patients and not only severe asthma and COPD patients. For difficult to manage asthma, systematic approaches exist $^{18,19}$ with positive effects on asthma control, quality of life, and exacerbation frequency. ${ }^{19}$ However, the results are only applicable for $<15 \%$ of all asthma patients. ${ }^{20}$ Care pathways for in-hospital management of exacerbated COPD exist, with positive effects on 30-day readmission rate. ${ }^{21}$ The present study shows that a systematic approach seems to be beneficial for all patients with asthma as well as COPD referred to secondary care pulmonology. However, we based our conclusions on a limited number of patients who were evaluated with the systematic approach. However, there were no differences in baseline characteristics compared with the usual care group that consisted of 103 patients. A limitation of the present study is the possibility of bias during the selection process whether patients were evaluated with the systematic approach or with usual care. This could have led to patients with less severe disease in the systematic approach since patients with acute complaints were evaluated at the outpatient clinic for acute respiratory complaints. However, there were no differences in the number of days between referral and first visit, suggesting that a limited number of patients within the usual care group were referred for acute respiratory complaints. Another limitation is that the data were retrieved from only one university hospital. This could have resulted in more complex disease combined with comorbidities. Consequently, the results could be less generalizable to local hospitals. However, in this study only patients who were referred by the GP were included and those who were referred for second or third opinion were excluded. A third limitation is that patients who were evaluated with the systematic approach were evaluated by a limited number of pulmonologists, whereas the patients who received usual care were seen by multiple pulmonologists of the department. We do not think this affected the results, since all patients in the systematic approach group were systematically evaluated and the pulmonologist could not influence the standard diagnostic tests, whereas in the usual care group, the diversity of physicians reduces the possibility of a physician-dependent preference for diagnostic work-up.

Giving the results of the present study, we recommend the use of systematic approaches that direct physicians to register lifestyle advices, symptoms, and individual care plans in daily practice, with a limited number of standard diagnostic tests. We recommend prospective evaluation of the impact of this systematic approach on disease control, quality of life, lifestyle changes, and costs.

\section{Conclusion}

A predefined systematic approach in combination with an optional internet-based self-management support system is useful in clinical practice. Since there were no differences in the final diagnoses, this suggests that not all tests that were included in the systematic approach are regularly needed in the diagnostic work-up. The outcomes suggest that a designated systematic approach stimulates physicians to record lifestyle advices, symptoms, and individual care plans. Subsequently, this approach could increase the number of 
patients referred back to primary care, according to national healthcare guidelines in the Netherlands.

\section{Abbreviations}

CT, computed tomography; DLCO, diffusing capacity for carbon monoxide; ECG, electrocardiography; $\mathrm{Fe}_{\mathrm{NO}}$, fractional exhaled nitric oxide; GOLD, Global Initiative for Chronic Obstructive Lung Disease; GP, general practitioner; ICS, inhaled corticosteroids; LABA, long-acting beta2agonist; LAMA, long-acting muscarinic-antagonist; LTRA, leukotriene receptor antagonist; LUMC, Leiden University Medical Center; SABA, short-acting beta2-agonist; SAMA, short-acting muscarinic-antagonist.

\section{Data sharing statement}

The data set used and analyzed during the current study are available from the corresponding author on reasonable request. Before this request, users should get permission from the medical ethical committee of the LUMC.

\section{Acknowledgments}

The authors would like to thank $\mathrm{C}$ van der Meij (nurse practitioner, Department of Pulmonology, LUMC, Leiden, the Netherlands) for the clinical assessment of patients, Dr JK Sont (associate professor, Department of Medical Decision Making, LUMC, Leiden, the Netherlands) for the availability of PatientCoach, Dr JB Snoeck-Stroband (GP, Department of Medical Decision Making, LUMC, Leiden, the Netherlands) for a GP's view on the diagnostic approach, and CE Schmidt (Advisor eHealth and Innovation, Department of Quality and Patient Safety, LUMC, Leiden, the Netherlands) for communication between different departments of the LUMC. This study was supported by Novartis with an unrestricted research grant.

\section{Author contributions}

Design of the study: ARvB, TNB, MJK, AMS, NHC, and CT. Data analysis: ARvB and TSW. Data interpretation: ARvB, TSW, TNB, MJK, AMS, NHC, and CT. Drafting the manuscript: ARvB, TSW, and TNB. Revision of the manuscript: ARvB, MJK, AMS, NHC, and CT. All authors contributed toward data analysis, drafting and revising the paper and agree to be accountable for all aspects of the work.

\section{Disclosure}

The authors report no conflicts of interest in this work.

\section{References}

1. Global Initiative for Asthma. Available from: http://ginasthma.org/. Accessed January 30, 2018.
2. Global Initiative for Chronic Obstructive Lung Disease. Available from: http://goldcopd.org/. Accessed January 20, 2018.

3. British Thoracic Society. British guideline on the management of asthma. Available from: https://www.brit-thoracic.org.uk/documentlibrary/clinical-information/asthma/btssign-asthma-guideline-2016/. Accessed March 27, 2018.

4. Campbell H, Hotchkiss R, Bradshaw N, Porteous M. Integrated care pathways. BMJ. 1998;316(7125):133-137.

5. van den Akker EF, van 't Hul AJ, Chavannes NH, et al. Development of an integral assessment approach of health status in patients with obstructive airway diseases: the CORONA study. Int J Chron Obstruct Pulmon Dis. 2015;10:2413-2422.

6. Kim MY, Lee SY, Jo EJ, et al. Feasibility of a smartphone application based action plan and monitoring in asthma. Asia Pac Allergy. 2016;6(3):174-180.

7. van Gaalen JL, Bakker MJ, van Bodegom-Vos L, et al. Implementation strategies of internet-based asthma self-management support in usual care. Study protocol for the IMPASSE cluster randomized trial. Implement Sci. 2012;7:113.

8. Peters JB, Daudey L, Heijdra YF, Molema J, Dekhuijzen PN, Vercoulen JH. Development of a battery of instruments for detailed measurement of health status in patients with COPD in routine care: the Nijmegen Clinical Screening Instrument. Qual Life Res. 2009;18(7): 901-912.

9. Juniper EF, O'Byrne PM, Guyatt GH, Ferrie PJ, King DR. Development and validation of a questionnaire to measure asthma control. Eur Respir J. 1999;14(4):902-907.

10. Kon SS, Dilaver D, Mittal M, et al. The Clinical COPD Questionnaire: response to pulmonary rehabilitation and minimal clinically important difference. Thorax. 2014;69(9):793-798.

11. Lange P, Halpin DM, O’Donnell DE, Macnee W. Diagnosis, assessment, and phenotyping of COPD: beyond $\mathrm{FEV}_{1}$. Int J Chron Obstruct Pulmon Dis. 2016;(11 Spec Iss):3-12.

12. NHG-Standaard COPD. 2015. Available from: https://www.nhg.org/ standaarden/volledig/nhg-standaard-copd. Accessed May 1, 2018.

13. Rotter T, Kinsman L, James E, et al. The effects of clinical pathways on professional practice, patient outcomes, length of stay, and hospital costs: cochrane systematic review and meta-analysis. Eval Health Prof. 2012;35(1):3-27.

14. de Veer AJ, Peeters JM, Brabers AE, Schellevis FG, Rademakers JJ, Francke AL. Determinants of the intention to use e-Health by community dwelling older people. BMC Health Serv Res. 2015;15:103.

15. Hofstede J, de Bie J, van Wijngaarden B, Heijmans M, Knowledge HM. Knowledge, use and attitude toward eHealth among patients with chronic lung diseases. Int J Med Inform. 2014;83(12):967-974.

16. Eysenbach G. The law of attrition. J Med Internet Res. 2005;7(1):e11.

17. van Gaalen JL, van Bodegom-Vos L, Bakker MJ, Snoeck-Stroband JB, Sont JK. Internet-based self-management support for adults with asthma: a qualitative study among patients, general practitioners and practice nurses on barriers to implementation. BMJ Open. 2016;6(8): e010809.

18. Honkoop PJ, Pinnock H, Kievits-Smeets RM, Sterk PJ, Dekhuijzen PN, In ' $t$ Veen JC. Adaptation of a difficult-to-manage asthma programme for implementation in the Dutch context: a modified e-Delphi. NPJ Prim Care Respir Med. 2017;27:16086.

19. Tay TR, Lee J, Radhakrishna N, et al. A structured approach to specialist-referred difficult asthma patients improves control of comorbidities and enhances asthma outcomes. J Allergy Clin Immunol Pract. 2017;5(4):e953:956-964.

20. Chipps BE, Zeiger RS, Dorenbaum A, et al. Assessment of asthma control and asthma exacerbations in the epidemiology and natural history of asthma: outcomes and treatment regimens (TENOR) observational cohort. Curr Respir Care Rep. 2012;1(4):259-269.

21. Vanhaecht K, Lodewijckx C, Sermeus W, et al. Impact of a care pathway for COPD on adherence to guidelines and hospital readmission: a cluster randomized trial. Int J Chron Obstruct Pulmon Dis. 2016;11: 2897-2908. 


\section{Supplementary materials}

Table SI Final diagnosis, phenotypes, and treatment plans in the diagnostic pathway compared to usual care

\begin{tabular}{|c|c|c|c|c|}
\hline Final diagnosis and treatment & $\begin{array}{l}\text { Total } \\
\text { group } \\
(\mathrm{N}=|| 7)\end{array}$ & $\begin{array}{l}\text { Usual care } \\
(\mathrm{N}=103)\end{array}$ & $\begin{array}{l}\text { Systematic } \\
\text { approach } \\
(\mathrm{N}=14)\end{array}$ & $\begin{array}{l}\text { Difference } \\
\text { (P-value) }\end{array}$ \\
\hline \multicolumn{5}{|l|}{ Final diagnosis } \\
\hline Asthma, n (\%) & $65(55.6)$ & $56(54.4)$ & $9(64.3)$ & 0.48 \\
\hline Phenotyped, n (\% yes) & $55(84.6)$ & $46(82.1)$ & $9(100)$ & 0.17 \\
\hline Allergic asthma, ${ }^{*} \mathrm{n}(\%$ yes $)$ & $43(66.2)$ & $35(62.5)$ & $8(88.9)$ & 0.12 \\
\hline Nonallergic asthma, ${ }^{*}$ (\% yes) & $10(15.4)$ & $9(16.1)$ & I (II.I) & 0.70 \\
\hline Late-onset, $*$ n (\% yes) & $6(9.2)$ & $6(10.7)$ & $0(0.0)$ & 0.30 \\
\hline With fixed airflow limitation,* n (\% yes) & $4(6.2)$ & $4(7.1)$ & $0(0.0)$ & 0.41 \\
\hline Asthma with obesity, ${ }^{*} \mathrm{n}$ (\% yes) & $0(0.0)$ & $0(0.0)$ & $0(0.0)$ & NA \\
\hline COPD, n (\%) & $29(24.8)$ & $27(26.2)$ & $2(14.3)$ & 0.33 \\
\hline Phenotyped, n (\% yes) & $17(58.6)$ & $16(59.3)$ & I (50.0) & 0.80 \\
\hline AAT deficiency, ${ }^{*}$ n (\% yes) & $0(0.0)$ & $0(0.0)$ & $0(0.0)$ & NA \\
\hline Emphysema/hyperinflation,* n (\% yes) & $6(20.7)$ & $6(22.2)$ & $0(0.0)$ & 0.45 \\
\hline Frequent exacerbations, ${ }^{*}$ n (\% yes) & II (37.9) & $10(37.0)$ & I $(50.0)$ & 0.72 \\
\hline Combination of asthma and COPD, $\mathrm{n}(\%)$ & I (0.9) & I ( $(1.0)$ & $0(0.0)$ & 0.71 \\
\hline Phenotyped, n (\% yes) & I (100) & $\mathrm{I}(100)$ & $0(0.0)$ & NA \\
\hline Other pulmonary disease, $\mathrm{n}$ (\% yes) & $5(4.3)$ & $4(3.9)$ & I (7.I) & 0.57 \\
\hline No pulmonary disease, $\mathrm{n}$ (\% yes) & II (9.4) & $10(9.7)$ & $\mathrm{I}(7.1)$ & 0.76 \\
\hline Obesity-related symptoms, n (\% yes) & $2(1.7)$ & $2(1.9)$ & $0(0.0)$ & 0.60 \\
\hline No diagnosis, $\mathrm{n}$ (\% yes) & $2(1.7)$ & $2(1.9)$ & $0(0.0)$ & 0.60 \\
\hline Lifestyle advices, $n$ (\% yes) & $43(36.8)$ & $30(29.1)$ & $13(92.9)$ & $<0.001$ \\
\hline Symptom scores, n (\% yes) & $36(30.8)$ & $22(21.4)$ & $14(100)$ & $<0.001$ \\
\hline Individual care plans, $\mathrm{n}$ (\% yes) & $16(13.7)$ & $8(7.8)$ & $8(57.1)$ & $<0.00$ I \\
\hline
\end{tabular}

Notes: *Patients could be categorized into more than one phenotype. Bold values indicate statistical significance.

Abbreviation: AAT, alpha I-antitrypsin.

Table S2 Time between referral, first visit, final diagnosis, latest diagnostic test, and referral back to primary care

\begin{tabular}{|c|c|c|c|c|}
\hline Time & $\begin{array}{l}\text { Total } \\
\text { group } \\
(\mathbf{N}=|| 7)\end{array}$ & $\begin{array}{l}\text { Usual care } \\
(\mathrm{N}=103)\end{array}$ & $\begin{array}{l}\text { Systematic } \\
\text { approach } \\
(\mathrm{N}=14)\end{array}$ & $\begin{array}{l}\text { Difference } \\
\text { (P-value) }\end{array}$ \\
\hline From referral to first visit in days (mean, SD) & $37.7(23.7)$ & $38.4(24.6)$ & $32.1(14.1)$ & 0.35 \\
\hline From first visit to final diagnosis in days (mean, SD) & $69.6(89.3)$ & $72.3(91.7)$ & $49.7(69.4)$ & 0.38 \\
\hline From first visit to latest diagnostic test in days (mean, SD) & $119.3(128.3)$ & $122.7(128.7)$ & $94.0(127.0)$ & 0.43 \\
\hline Referred back to primary care, $\mathrm{n}(\%)$ & $70(59.8)$ & $58(56.3)$ & $12(85.7)$ & 0.04 \\
\hline Time from first visit and referral to primary care in days (mean, SD) & $117.5(97.9)$ & II3.3 (96.0) & I 37.7 (I08.6) & 0.44 \\
\hline
\end{tabular}

Note: Bold values indicate statistical significance.

\section{Publish your work in this journal}

The International Journal of COPD is an international, peer-reviewed journal of therapeutics and pharmacology focusing on concise rapid reporting of clinical studies and reviews in COPD. Special focus is given to the pathophysiological processes underlying the disease, intervention programs, patient focused education, and self management protocols.

\section{Dovepress}

This journal is indexed on PubMed Central, MedLine and CAS. The manuscript management system is completely online and includes a very quick and fair peer-review system, which is all easy to use. Visit $\mathrm{http}: / /$ www.dovepress.com/testimonials.php to read real quotes from published authors. 